\title{
OpenFOAM custom solver for Flame Spray Pyrolysis simulation
}

\author{
Nadine Zandoná Rafagnim*1, Pedro Bianchi Neto${ }^{1}$, Udo Fritsching ${ }^{2}$, Dirceu Noriler ${ }^{1}$ \\ ${ }^{1}$ Department of Process Engineering - DEPro, University of Campinas, Brazil \\ ${ }^{2}$ Leibniz Institute for Materials Engineering - IWT, Bremen, Germany \\ ${ }^{*}$ Corresponding author email: nrafagnim@gmail.com
}

\begin{abstract}
Nanoparticles of inorganic materials can be efficiently produced through the Flame Spray Pyrolysis process. With validated mathematical models, CFD techniques can be useful for the scaleup of this process and design of new materials. Using CFD open source codes can give more certainty about the mathematical models and methods being applied, but they do not always offer a large variety of models for robust and stable simulations as commercial codes do. In this paper, we propose a methodology to solve FSP ethanol flames through a custom OpenFOAM 8 based solver named simpleBuoyantSprayFoam. Its main features are the compressibility, the capability of solving the Lagrangian field interaction with the continuous phase, buoyancy effects which allow for air entrainment and the turbulent chemistry. Furthermore, the SIMPLE algorithm is used, and a steady solution is obtained in viable computational time. To ensure the solver consistency, three different operating conditions were tested, varying the ethanol flowrates, and the obtained results were compared with experimental data and commercial code results. Good agreement was shown amongst all results, which highlights the possibilities of using free open source codes for complex CFD simulation.
\end{abstract}

\section{Keywords}

open source; combustion, reactive flow; nanoparticles; CFD.

\section{Introduction}

Nanoparticles of metallic oxides attract attention due to their special characteristics such as high ratio between surface area and volume, chemical stability, catalytic properties, and the mechanical and thermal resistance. Their applicability ranges from paints and polishing sludges to antimicrobials drugs and fuel cell devices $(1 ; 2)$. For their many applications, the size of nanoparticle cluster is usually an essential parameter (3). For metallic oxide nanoparticles synthesis, the Flame Spray Pyrolysis (FSP) is an interesting technique which allows for the efficient and economical production with high purity and narrow size distribution in the range from 1 to $200 \mathrm{~nm}$ (4). In this process, a low cost metallic precursor is dissolved in a liquid fuel (i.e. organic solvent) and is supplied into the reactor through a nozzle. A dispersion gas is responsible for the atomization of the liquid into fine droplets, which will evaporate and oxidise, generating an oversaturated region where nucleation takes place. The nuclei then undergo different processes of growth, including, for instance, agglomeration, and sintering, along the spray's trajectory. A scheme of the FSP process is shown in Figure 1.

The scaleup of the FSP process and its adaptation for the direct manufacture of functional nanostructured materials require the development of tools that integrate mathematical modeling and numerical simulations. Such tools make it possible to predict the main phenomena involved in the process, such as the chemistry-turbulence integration, the formation, and evolution of the spray, the heat and mass transfer due to evaporation and radiation, the nucleation and growth of the nanoparticles, among others. Simulations based on valid numerical models are useful to reduce the number of physical experiments (and associated costs). The numerical prediction of these phenomena, nonetheless, is complex and requires deep physical, chemical and thermodynamical knowledge of the process. 
Numerical investigations of FSP have proven to be able to capture diverse aspects of physical-chemical phenomena occurring at different time and space scales. Computational Fluid Dynamics (CFD) has been applied to several studies in the investigation of the FSP process, and good results have been observed $(5 ; 6 ; 7 ; 8)$. Numerical research such as these are usually focused on the implementation of complex and coupled physical models that aim at better describing the processes they want to study. The cost of commercial codes, however, may be an impediment to the development of research, not only in this field. Hence, the adoption of open source codes is a trend in the scientific community. In addition,

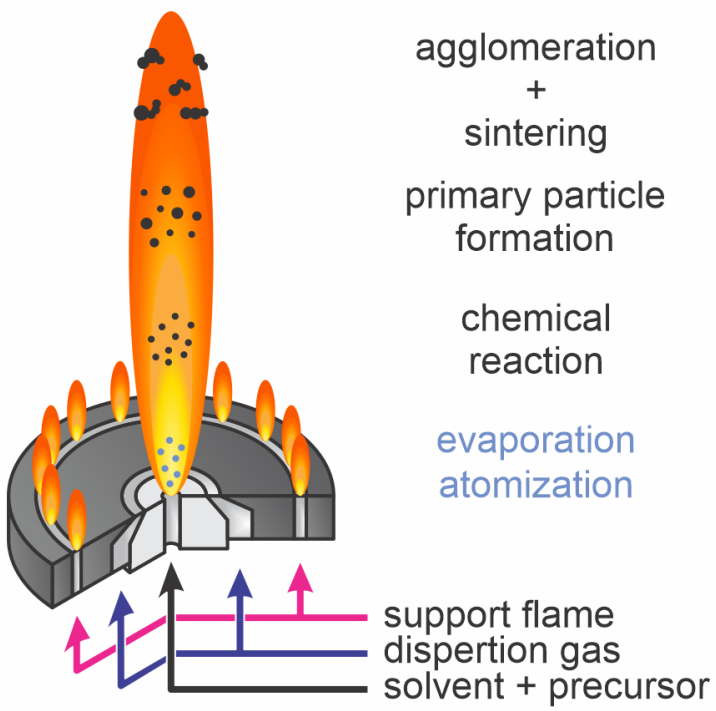

Figure 1. Scheme of the FSP process. codes such as OpenFOAM allow for the complete control over the employed models and the numerical methods, which enhances the quality of the work in numerical fields. A validated model can ultimately be used for industrial applications (9) at much smaller costs.

In this study, an OpenFOAM 8 custom solver was developed aiming at the FSP process simulation. The solver is capable of taking the main physical aspects of the process into consideration, such as buoyancy, turbulent chemistry interaction, compressibility and Lagrangian field interaction with the continuous phase. It was based on the sprayFoam solver, and it was modified to include buoyancy effects and to make it steady using the SIMPLE algorithm for pressurevelocity coupling. The custom solver was named simpleBuoyantSprayFoam. Ethanol flames were simulated and the obtained numerical results were compared for both OpenFOAM and ANSYS Fluent 14.5, commercial code which has proven to accurately describe the experimental FSP results in previous works $(10 ; 7)$. Experimental data for radial and axial temperature are also available and were used for validation purpose (11).

\section{Mathematical Modeling, Solver Description and Methodology}

Following (12), a compressible steady-state two-phase Euler-Lagrange model was chosen to describe the continuous gas phase and the discrete liquid droplets, respectively. The $k-\omega \mathrm{SST}$ model was used to describe the turbulence and a two-way coupling with turbulent dispersion $(14 ; 18)$ between the gas and liquid phases was considered. The mass and heat interphase transfers of the vaporizing droplets were accounted by the (15) correlation. Radiation was described by P-1 model, considering a grey gas model. For the reactive flow, fuel and solvent oxidation reactions were considered, with a four-step mechanism for methane combustion (16) and a single step mechanism for ethanol (17). The Eddy Dissipation Concept (EDC) model was used to describe the interaction between turbulence and chemistry, assuming that the reactions occur in the fine scale turbulent structures, where the reactants are homogeneously mixed (10). Care was taken to use a numerical methodology as similar as possible for both Fluent and OpenFOAM. Therefore, the sprayFoam solver was modified to account for buoyancy effects and to provide steady-state solutions. This custom solver was named simpleBuoyantSprayFoam. Another important modification was made on the OpenFOAM 8 stochastic dispersion model to make it more similar to Fluent's Discrete Random Walk (DRW), which was used in previous works with good representation of droplet behavior $(12 ; 13)$. Differences are observed in the calculation of fluctuating velocity term as well as in the fluctuation period. For DRW, the particle- 
eddy interaction time is the smaller value between the eddy crossing time and the eddy lifetime, the first calculated as a function of the particle relaxation time and the second as a function of $k$ and $\epsilon$. In stochastic dispersion model the particle-eddy interaction time is the smaller between two terms related to $k$ and $\epsilon$ and particle relaxation time effects are not considered. Regarding the fluctuating velocity, in the stochastic model an extra random vector is used to account for the turbulence spatial randomness (more details are available in (18)). This extra term was removed in the OpenFOAM model so the fluctuating velocity could be calculated in the same way in both solvers. For now, modifications in particle-eddy interaction time were not made because they would require extensive changes in the code.

Pressure-based solvers were used for the solution of the gas phase in both programs and the SIMPLE algorithm was used for pressure-velocity coupling. Second order interpolation schemes were used for all variables interpolation (second-order upwind in Fluent and linear in OpenFOAM), except for turbulence variables $k$ and $\omega$ (first order upwind in both solvers).

The main boundary conditions are handled similarly in Fluent and OpenFOAM. For the pilot flame and oxygen inlet regions, mass flow inlets were considered, so a mass flow rate was specified. The walls are treated with no slip boundary conditions. In the air entrainment region, total pressure is specified for the backflow in both solvers (19). For the outflow Fluent will consider the specified value as the static pressure value (20), and in OpenFOAM it was observed that setting the total pressure boundary condition at the top caused backflow recirculation, so a zero gradient boundary condition for pressure was imposed on this patch.

The thermophysical parameters are calculated by JANAF thermodynamic database (21) in OpenFOAM and by piecewise polynomials in Fluent, and viscosities were assumed to be constant for all species. For chemistry solution, direct integration with DASAC solver (22) was used in Fluent, whereas in OpenFOAM the Eulerlmplicit method is employed with activated equilibriumRateLimiter and cTauChem equals to 0.25 . The simulations took about 4 days with OpenFOAM and about a week with Fluent using 28 Intel Xeon E5-2680 2.4 GHz cores.

A 3D domain was simulated with a mesh of about 1.6 million hexahedral elements. Ethanol was injected at 3,5 or $7 \mathrm{~mL} / \mathrm{min}$ through a conical surface above the nozzle. The droplets are dispersed by pure $\mathrm{O}_{2}(5 \mathrm{~L} / \mathrm{min})$ fed at a constant pressure drop of 1.5 bar at the nozzle tip. The spray flames were ignited and stabilized with a premixed support flame of $\mathrm{CH}_{4}(1.5 \mathrm{~L} / \mathrm{min})$ and $\mathrm{O}_{2}(3.2 \mathrm{~L} / \mathrm{min})$. Figure 2 shows the geometry, mesh and boundary regions. In the results section, cases are named after their ethanol and oxygen feed rates, e.g. 3_5 means $3 \mathrm{~mL} / \mathrm{min}$ of ethanol and $5 \mathrm{~L} / \mathrm{min}$ of oxygen.

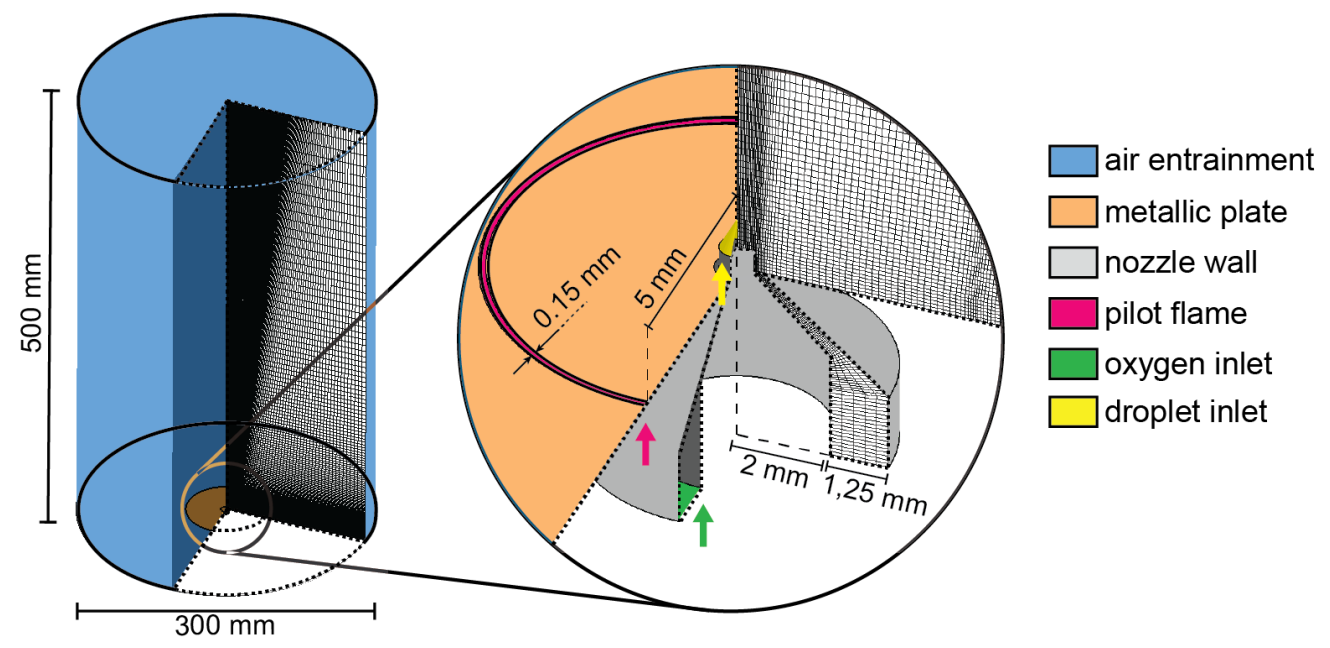

Figure 2. Geometry, mesh and main boundary conditions employed in this study. 


\section{Results and discussion}

In this section, results of velocity, temperature and ethanol profiles are shown and compared for both Fluent and OpenFOAM. Experimental data is also available for temperature, so it is possible to access which models better describe the involved phenomena. To evaluate the effect of the modification made on the dispersion model, Figure 3 shows axial temperature results for cases with $5 \mathrm{~mL} / \mathrm{min}$ of ethanol. Numerical results of Fluent (using the DRW model), OpenFOAM with the standard Stochastic dispersion model and OpenFOAM with the modified dispersion model are presented. Fluent's results are closer to the experimental points, followed by the OpenFOAM modified model, which was chosen for the OpenFOAM simulations presented hereafter.

Figure 4 shows axial velocities profiles at 100, 200 and $300 \mathrm{~mm}$ height above the burner (HAB). The velocities increase with the liquid flowrates, which is expected due to the higher temperatures associated with more fuel combustion and also higher evaporation rates, causing the liquid phase to evaporate and expand.

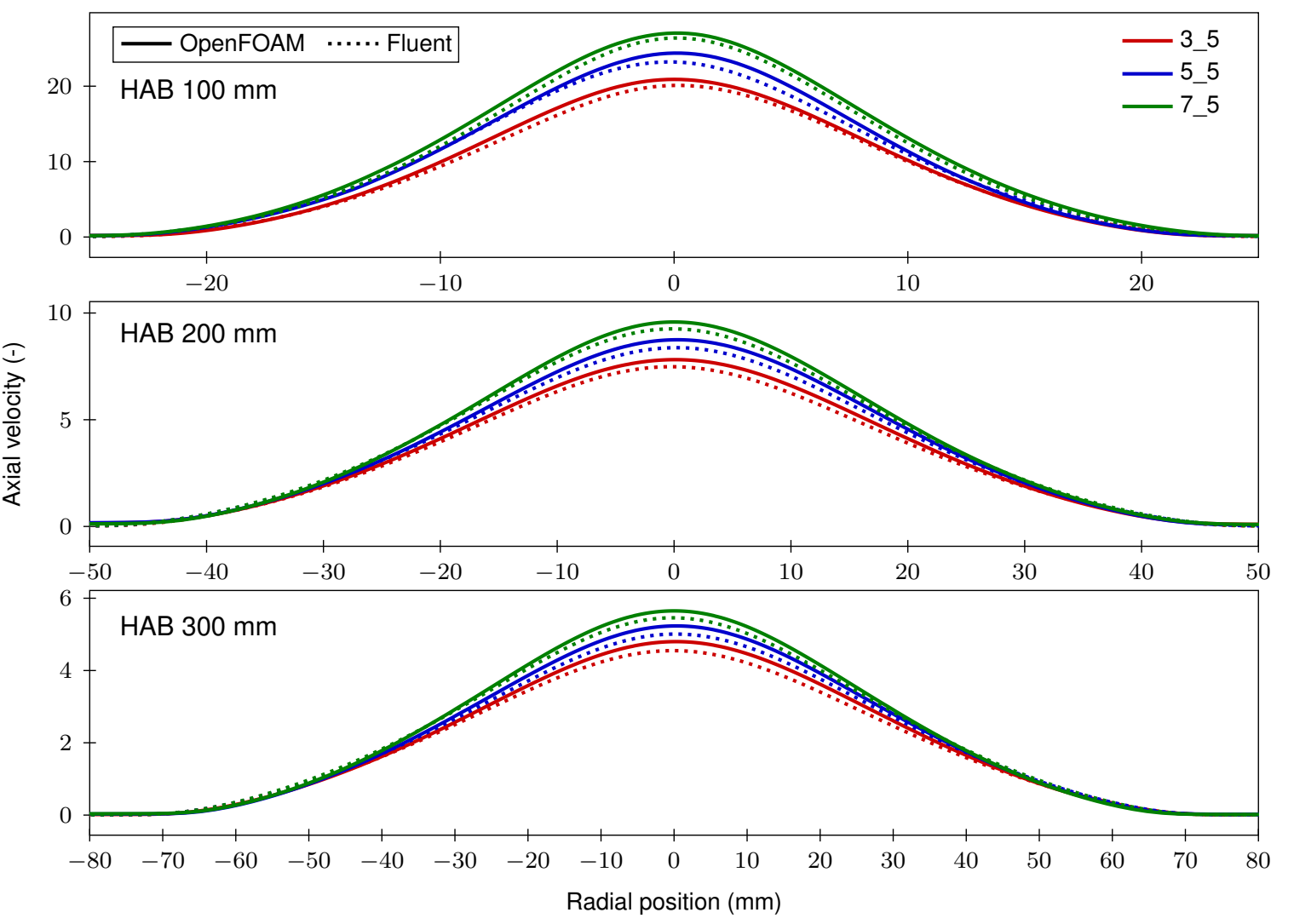

Figure 4. Axial velocities plotted against the radial coordinate in three different heights above burner (HAB) for all simulated cases.

These higher temperatures are responsible for expanding and accelerating the gas, but as the external cold air is drawn to the reaction zone as result of the buoyant upward flow of the 
hot gases, these velocities tend to decrease. This behavior is well described in both solvers results, but in general the velocities predicted by OpenFOAM are higher in central regions, and the maximum relative errors are about 6.4, 5.8 and $6.3(\mathrm{~m} / \mathrm{s}) \%$ for 100,200 and $300 \mathrm{~mm}$ above the burner, respectively.

Temperature contours at a central plane and their respective flame heights (region with temperature above $1500 \mathrm{~K}$ ) are presented in Figure 5 . The observed behavior is consistent with what was described previously for the velocity, where higher temperatures are reached in higher regions of the reactor with OpenFOAM and, therefore, higher velocities are also observed. The OpenFOAM flames are about 7.8, 6.7 and $6.6 \mathrm{~mm} \%$ higher than the Fluent ones for 3, 5 and $7 \mathrm{~mL} / \mathrm{min}$ of ethanol, respectively.

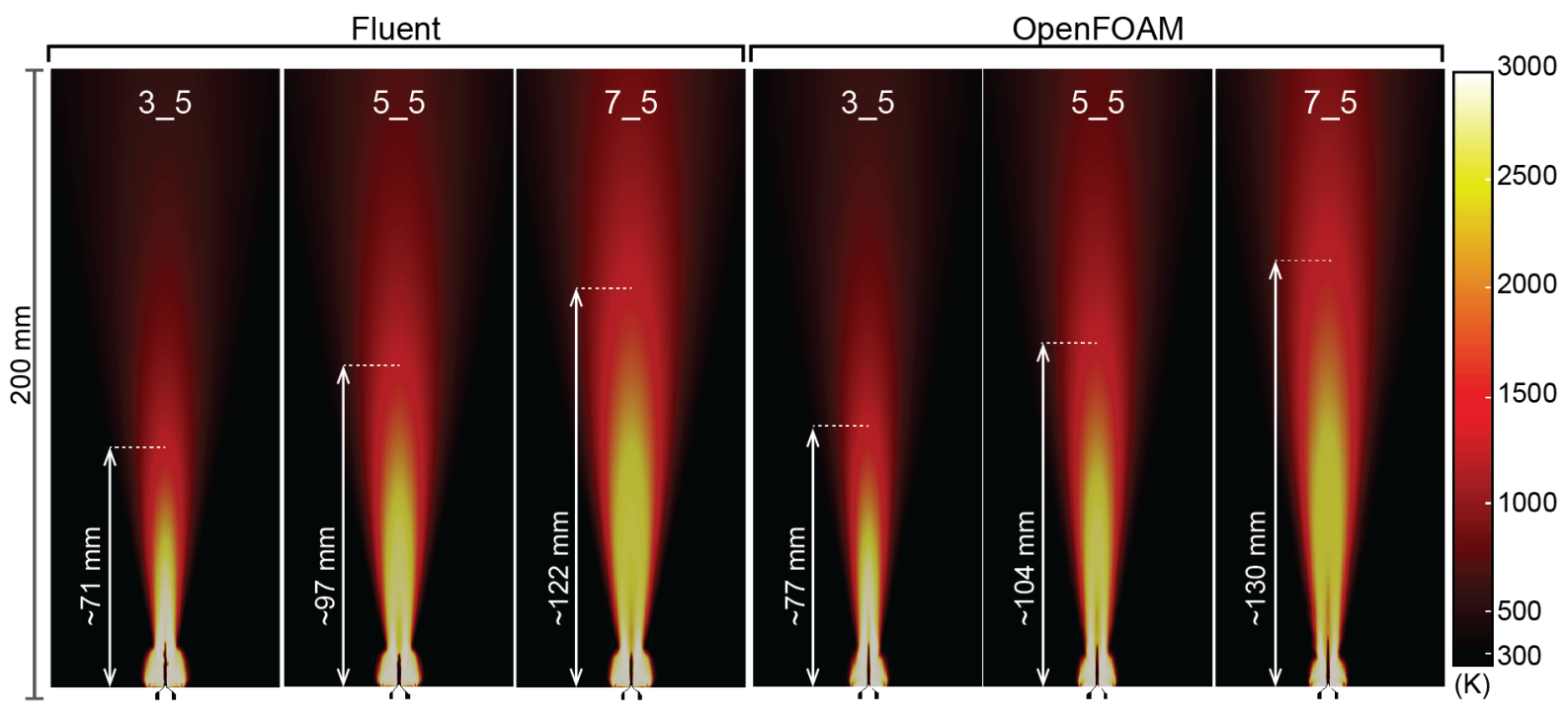

Figure 5. Temperature fields with their respective flame heights.

It can also be observed in Figure 5 that the flames obtained with OpenFOAM are narrower than the Fluent ones. This behavior, when analysed alongside Figure 6, shows that there is a

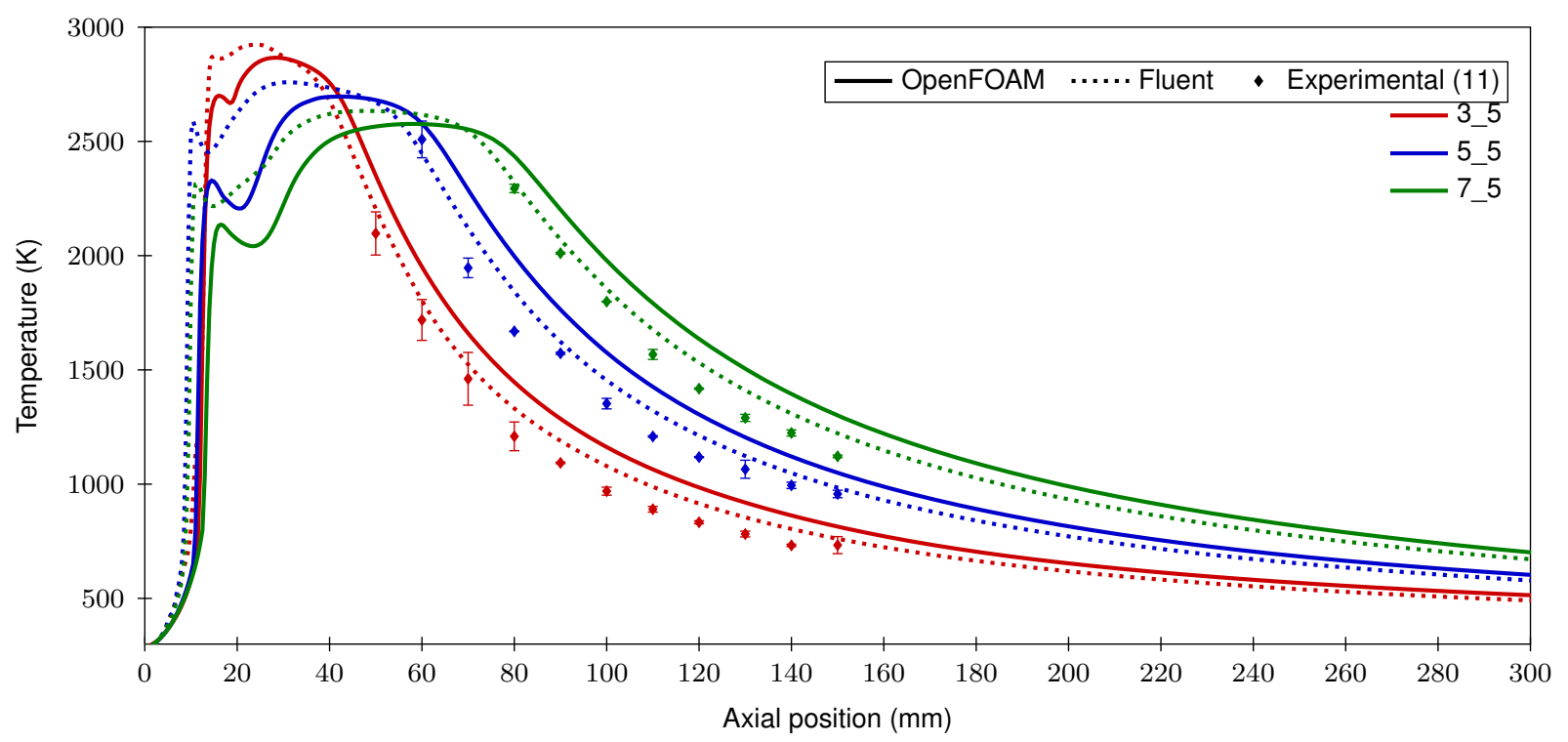

Figure 6. Temperature profiles along the center line of the reactor

delay in the ethanol combustion in OpenFOAM when compared to Fluent, (which once again is sustained by the higher velocities observed in central region). Higher temperature peaks 
are reached with Fluent right at the bottom of the reactor, and then the temperature decreases faster. Qualitatively both solvers show a similar behavior, but the results obtained with Fluent are closer to the experimental data.

When the different ethanol operating conditions are compared in Figure 6, it can be seen that higher peaks are reached with smaller ethanol flowrates. This happens because of the smaller amount of cold liquid injected, so less heat has to be transferred to evaporate the liquid phase, and consequently combustion is responsible for heating the reactor right at its lower regions. This effect and the presence of less fuel cause the temperature to drop faster for operating conditions with less ethanol, and from about $80 \mathrm{~mm}$ above the burner higher temperatures are always associated with higher fuel loads.

Temperature at lines 100, 200 and $300 \mathrm{~mm}$ above the burner level are presented in Figure 7. In general there is an inversion and the Fluent temperatures are higher on external regions and OpenFOAM temperatures are higher at central regions, with an exception for the $300 \mathrm{~mm}$ height. This also corroborates that OpenFOAM produces narrower flames, with smaller temperatures in external regions and high temperatures at the center.

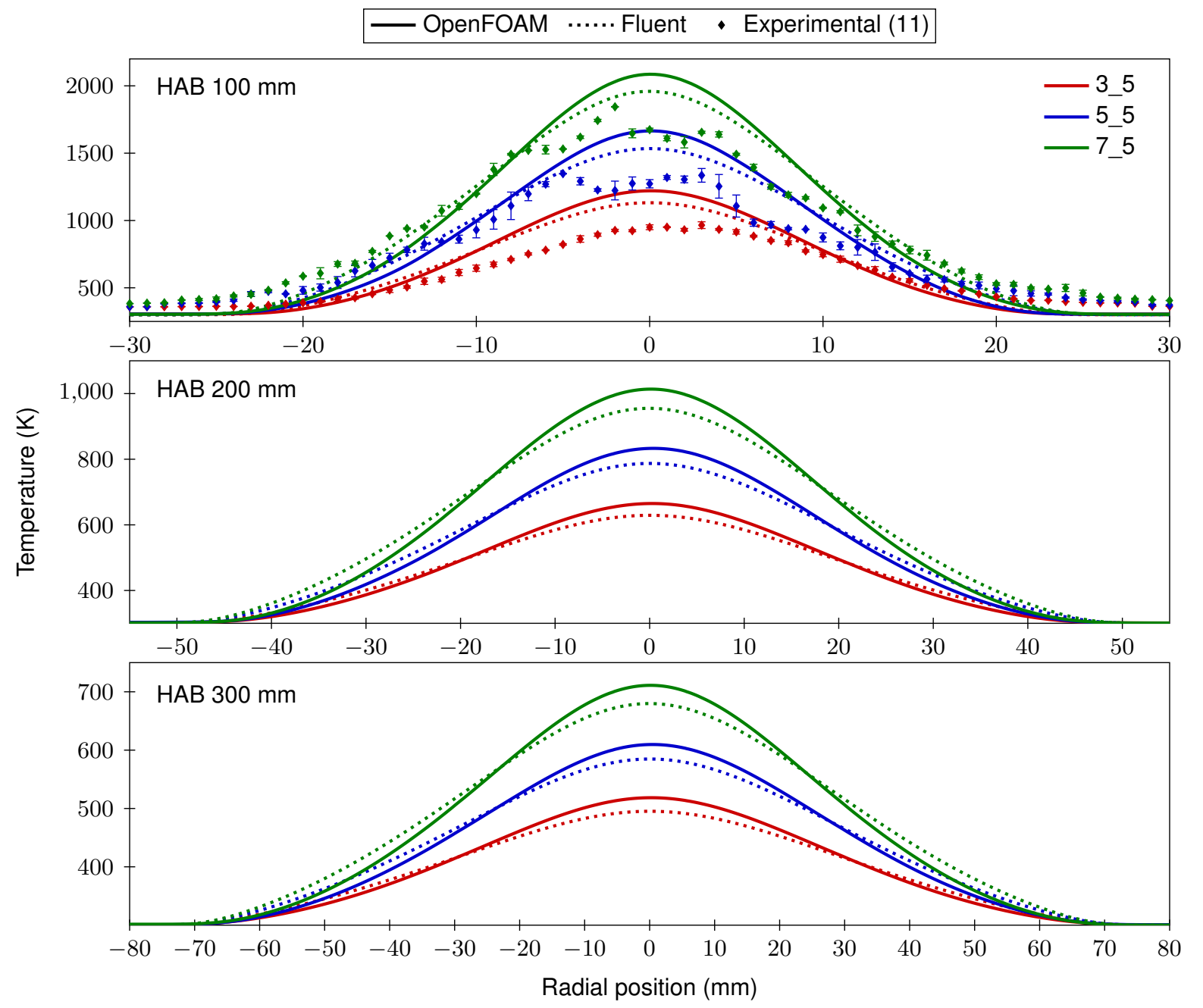

Figure 7. Temperature plotted against the radial coordinate in three different heights above burner (HAB) for all simulated cases.

Figure 8 shows ethanol mass fraction contours at the reactor center. With higher fuel loads, the ethanol mass fractions increase and reach higher positions, which is also associated with the higher velocities observed with the different fuel loads. The profiles obtained with OpenFOAM are more regular than those obtained with Fluent, which points to the remaining differences in the lagrangian model. The different pattern obtained with Fluent are very likely associated with 
the dispersion model used, which is responsible for incorporate the turbulence random effects on the particle motion. As indicated in Figure 3, the dispersion model significantly alters the obtained results, and it is very likely responsible for differences observed in temperature and velocity as well. Because the results obtained with Fluent are closer to the available experimental data, matching the models implemented in the commercial solver could help to improve the OpenFOAM solution.

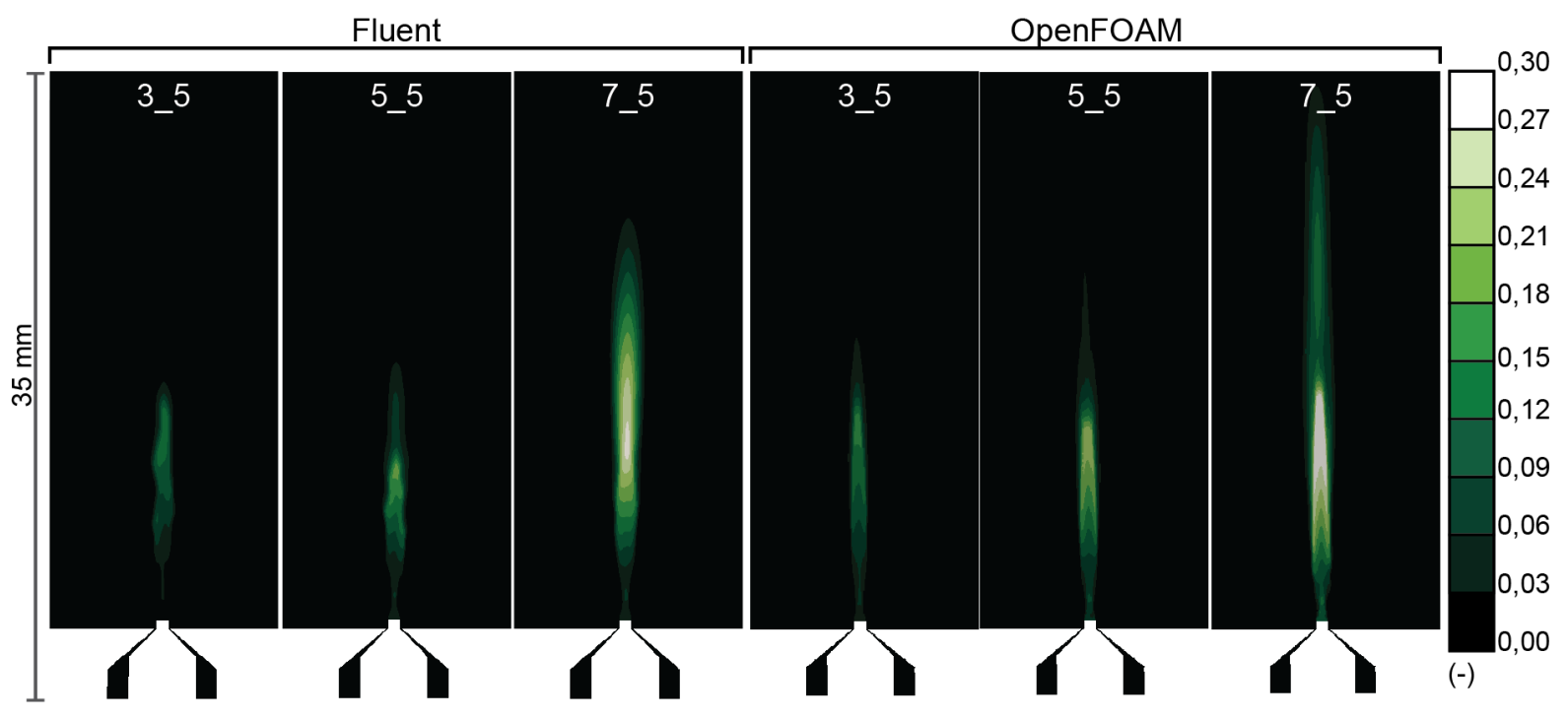

Figure 8. Ethanol mass fraction profiles.

\section{Conclusions}

The simpleBuoyantSprayFoam solver was developed based on the sprayFoam solver for simulating the FSP process. It incorporates buoyancy, compressibility, turbulent chemistry interaction and lagrangian field interaction with the continuous phase effects. Further than the solver development, an important modification in the stochastic turbulent dispersion model available with OpenFOAM 8 was made.

Through the comparison with ANSYS Fluent 14.5 results and experimental temperature data, good agreement was found, which indicates that simulating complex processes such as the FSP using open and free codes such as OpenFOAM is possible and can provide reliable results. The observed differences between the commercial and the developed solvers are associated with the lagrangian modeling, namely the dispersion model. Further than matching these models, increasing the turbulence resolution using Large Eddy Simulation models could also increase the solution accuracy and approximate the numerical and experimental data. Future works must also focus on including the solid nanoparticle simulations, which can be addressed with a Population Balance Model.

\section{Acknowledgements}

This study received funding from National Council for Scientific and Technological Development (CNPq, Project ID: 140351/2020-5) and São Paulo Research Foundation - FAPESP (Projects IDs: $2017 / 04045-0$ and 2020/08502-9). 


\section{References}

[1] Mehta, M., Raman, V., Fox, R. O., 2013, Chemical Engineering Science, 104, pp. 10031018.

[2] Wegner, K., Pratsinis, S. E., 2005, Powder Technology, 150, pp. 117-122.

[3] Torabmostaedi, Ho. and Zhang, T., 2014, Journal of Thermal Spray Technology, 23, pp. 1478-1492.

[4] Mädler, L., Kammler, H. K., Mueller, R., Pratsinis, S. E., 2002, Journal of Aerosol Science, 33, pp. 369-389.

[5] Gröhn, A. J., Buesser, B., Jokiniemi, J. K., Pratsinis, S. E., 2011, Industrial and Engineering Chemistry Research, 50, pp. 3159-3168.

[6] Olivas-Martinez, M., Sohn, H. Y., Jang, H. D., Rhee, K. I., 2015, Journal of Nanoparticle Research, 17, pp. 1-17.

[7] Bianchi Neto, P., Buss, L., Meierhofer, F., Meier, H. F., Fritsching, U., Noriler, D., 2020, Powder Technology, 370, pp. 116-128.

[8] Buss, L., Noriler, D., Fritsching, U., 2020, Flow, Turbulence and Combustion, 105.

[9] Jasak, H., 2009, International Journal of Naval Architecture and Ocean Engineering, 1, pp. 89-94.

[10] Bianchi Neto, P., Buss, L., Meierhofer, F., Meier, H. F., Fritsching, U., Noriler, D., 2018, Chemical Engineering and Processing - Process Intensification, 129, pp. 17-27.

[11] Bianchi Neto, P., 2017, "Análise numérica do crescimento de nanopartículs em reator de pirólis em spray flamejante usando balanço populacional bivariado" - master's thesis, 2017.

[12] Noriler, D., Rosebrock, C. D., Mädler, L., Meier, H. F., Fritsching, U., 2014, Atomization and Sprays, 24, pp. 495-524.

[13] Bianchi Neto, P., Buss, L., Meierhofer, F., Meier, H. F., Fritsching, U., Noriler, D., 2018, Powder Technology, 370, pp. 116-128.

[14] ANSYS, 2013, "ANSYS Fluent Theory Guide (section 16.2.2.1.2. The Discrete Random Walk Model)".

[15] Ranz, W. E., Marshall, W. R., 1952, Evaporation from drops, 48, pp. 141-146.

[16] Jones, W. P., Lindstedt, R. P., 1988, Combustion and Flame, 73, pp. 233-249.

[17] Westbrook, C. K., Dryer, F. L., 1981, Combustion Science and Technology, 27, pp. 31-43.

[18] Greifzu, F., Kratzsch, C., Forgber, T., Lindner, F., Schwarze, R., 2016, Engineering Applications of Computational Fluid Mechanics, 10, pp. 30-43.

[19] OpenFOAM: User Guide: Total Pressure openfoam.com/documentation/guides/latest/ doc/guide-bcs-inlet-outlet-total-pressure.html

[20] ANSYS, 2013, "ANSYS Fluent User's Guide (section 6.3.8.1.3 - Defining Backflow Conditions)".

[21] NIST-JANAF Thermochemical Tables janaf.nist.gov.

[22] Caraccotsios, M., Stewart, W.E., 1985, Computers \& Chemical Engineering, 9 (4), pp. 359-365. 